2. A. E. Mayer, Grösste Polygone mit gegebenen Seitenvektoren, Comment. Math. Helv. vol. 10 (1938) pp. 288-301.

3. H. Hadwiger, Über eine Mittelwertformel für Richtungsfunktionale im Vektorraum und einige Anwendungen, J. Reine Angew. Math. vol. 185 (1943) pp. 241-252.

4. R. Courant and E. J. McShane, Differential and integral calculus, Interscience, 1947, vol. 2, pp. 303-304.

Los Angeles City College

\title{
ON SETS OF CONSTANT WIDTH
}

\section{GERALD FREILICH}

Sets of constant width in $E^{n}$ ( $E^{n}$ will denote Euclidean $n$-space) form an important subset of the set of all convex sets in $E^{n}$. For example, the Hausdorff $k$ measure $(0<k \leqq n)$ of a subset of $E^{n}$ is usually defined in terms of coverings of this set by arbitrary sets. However, because of the property that an arbitrary set is contained in a set of constant width of the same diameter, it is possible to refine this definition so that we need only consider coverings by sets of constant width. Unfortunately one cannot further refine the definition of Hausdorff measure, say to coverings by spheres, since it is not true that an arbitrary set is contained in a sphere of the same diameter. Thus in computing Hausdorff measures, the problem arises to find properties of sets of constant width that are analogous to those of spheres. It is the purpose of this paper to prove such a property; namely we establish a uniformity condition on sets of constant width (which incidentally does not hold for arbitrary convex sets). Roughly speaking, this uniformity condition states that parallel crosssections of a set of constant width vary continuously and that the variation for sets of given diameter $d$ can be characterized independent of the particular set. Though the motivation given above for theorems of this type arises from measure theory, I think that the results are interesting from a purely geometric point of view.

For the properties of convex sets and, in particular, sets of constant width, that will be used in this paper, the reader is referred to Theorie der konvexen Körper by J. Bonnesen and W. Fenchel, Ergebnisse der Mathematik, vol. 3, part 1, 1934. We shall in addition use the following notation:

As usual, the symbol $\rightarrow$ will denote implication between sen-

Received by the editors August 3, 1949 and, in revised form, October 25, 1949. 
tences. Also Lebesgue $n$ measure over $E^{n}$ will be denoted by $\mathcal{L}_{n}$, and if $A$ is a subset of $E^{n}$, then

$$
\int_{A} f(x) d \mathcal{L}_{n} x
$$

will stand for the (Lebesgue) integral of $f$ over $A$ with respect to the measure $\mathcal{L}_{n}$. The remainder of the notation will be standard. Thus for example, if $c$ is a subset of $E^{2}$, then

$$
\mathcal{L}_{1}\left(E^{1} \cap \underset{z}{E}[(1, z) \in c]\right)
$$

will denote the one-dimensional Lebesgue measure of the set of all points $z$ of $E^{1}$ for which $(1, z)$ lies in $c$.

Definition. If $c$ is a set of constant width in $E^{n}$, and $k$ is a positive integer less than $n$, then we define the function $g_{c}^{k}$ on $E^{n-k}$ to $E^{1}$ (the reals) by the formula

$$
g_{c}^{k}(x)=\mathcal{L}_{k}\left(E^{k} \cap \underset{z}{E}\left[\left(x_{1}, \cdots, x_{n-k}, z_{1}, \cdots, z_{k}\right) \in c\right]\right),
$$

for $x \in E^{n-k}$.

Intuitively, $g_{c}^{k}(x)$ can be thought of as the measure of a $k$-dimensional cross-section of the set $c$.

We now establish our uniformity conditions on sets of constant width, Theorem 1 below proving it for one-dimensional cross sections, and Theorem 2 proving it for higher-dimensional cross-sections.

THEOREM 1. If $c$ is a set in $E^{n}$ of constant width, diam $c=d$, and $\delta>0$, then

$\left(x \in E^{n-1}, y \in E^{n-1},|x-y| \leqq \delta\right) \rightarrow\left(\left|g_{c}^{1}(x)-g_{c}^{1}(y)\right| \leqq 2^{3 / 2} \cdot(\delta d)^{1 / 2}\right)$.

Proof. Let $\bar{c}$ be the projection of $c$ into the first $(n-1)$ coordinates, that is,

$$
\bar{c}=E^{n-1} \cap \underset{x}{E}\left[\left(x_{1}, \cdots, x_{n-1}, t\right) \in c \text {, for some } t,-\infty<t<\infty\right],
$$

and let $\beta$ be the boundary of $\bar{c}$. Then $\bar{c}$ is a set of constant width in $E^{n-1}$.

The following fact is well known:

If $h$ is a convex function on the interval

$$
\left(E^{1} \cap \underset{x}{E}[a \leqq x \leqq b]\right)
$$

to $E^{1}$ and $\lambda>0$, then either 


$$
\sup _{|x-a| \leqq \lambda}|h(x)-h(a)|=\sup _{|x-y| \leqq \lambda}|h(x)-h(y)|
$$

or

$$
\sup _{|x-b| \leqq \lambda}|h(x)-h(b)|=\sup _{|x-y| \leqq \lambda}|h(x)-h(y)| .
$$

Suppose now that $x \in \bar{c}, y \in \bar{c}$, and $0<|x-y| \leqq \delta$. Then the line determined by $x$ and $y$ intersects $\beta$ in two points; thus there exist $a \in \bar{c}, b \in \bar{c}$ such that

$$
\underset{x}{E}[x=a \text { or } x=b]=(\beta \cap \underset{z}{E}[z=y+t(x-y),-\infty<t<\infty]) .
$$

Since the function $g_{c}^{1}$ confined to the set

$$
(\bar{c} \cap \underset{z}{E}[z=a+t(b-a), 0 \leqq t \leqq 1])
$$

is a convex function, we conclude that either

$$
\left|g_{c}^{1}(x)-g_{c}^{1}(y)\right| \leqq \sup _{|z-a| \leqq \delta}\left|g_{c}^{1}(z)-g_{c}^{1}(a)\right|,
$$

or

$$
\left|g_{c}^{1}(x)-g_{c}^{1}(y)\right| \leqq \sup _{|z-b| \leqq \delta}\left|g_{c}^{1}(z)-g_{c}^{1}(b)\right| .
$$

Hence it follows that

$$
\sup _{x \in \bar{c}, y \in \bar{c}_{\bar{c}}|x-y| \leqq \delta}\left|g_{c}^{1}(x)-g_{c}^{1}(y)\right|=\sup _{x \in \beta, y \in \bar{c}_{\bar{c},|x-y| \leqq \delta}}\left|g_{c}^{1}(x)-g_{c}^{1}(y)\right| .
$$

Now let $x \in \beta, y \in \bar{c}, 0<|x-y| \leqq \delta$. Since $x$ is a boundary point of $\bar{c}$, it follows that there is a unique number $t$ for which $\left(x_{1}, \cdots, x_{n-1}, t\right)$ $\in c$ and also that there exists an $(n-2)$-dimensional supporting plane $P \subset E^{n-1}$ of $\bar{c}$ through $x$. Let $P^{\prime}$ be the line in $E^{n}$ determined by $\left(x_{1}, \cdots, x_{n-1}, 0\right)$ and $\left(x_{1}, \cdots, x_{n-1}, 1\right)$. Then, considering $P \subset E^{n-1}$ as imbedded in $E^{n}, P$ and $P^{\prime}$ obviously determine an $(n-1)$-dimensional supporting plane to $c$ through $\left(x_{1}, \cdots, x_{n-1}, t\right)$. Since $c$ is a set of constant width, we may choose $x^{\prime} \in \bar{c}$ such that $\left|x-x^{\prime}\right|=d$, and such that $\left(x_{1}^{\prime}, \cdots, x_{n-1}^{\prime}, t\right) \in c$. Hence

$$
c \subset\left(E^{n} \cap \underset{z}{E}\left[\left|z-\left(x_{1}^{\prime}, \cdots, x_{n-1}^{\prime}, t\right)\right| \leqq d\right]\right)=S .
$$

Now the intersection of $S$ with the 2-dimensional plane determined by $\left(x_{1}, \cdots, x_{n-1}, 0\right),\left(y_{1}, \cdots, y_{n-1}, 0\right)$, and $\left(y_{1}, \cdots, y_{n-1}, 1\right)$ is a disc of radius $r \leqq d$, whose circumference passes through $\left(x_{1}, \cdots\right.$, 
1951]

ON SETS OF CONSTANT WIDTH

95

$\left.x_{n-1}, t\right)$, and such that the $n$th coordinate of its center is $t$. Since the length of the vertical chord of this disc passing through $\left(y_{1}, \cdots\right.$, $\left.y_{n-1}, t\right)$ is $2\left(r^{2}-(r-|x-y|)^{2}\right)^{1 / 2}=2\left(2 r \cdot|x-y|-|x-y|^{2}\right)^{1 / 2}$, and since $g_{c}^{1}(x)=0$, we conclude that

$$
\left|g_{c}^{1}(x)-g_{c}^{1}(y)\right|=g_{c}^{1}(y) \leqq 2\left(2 r \cdot|x-y|-|x-y|^{2}\right)^{1 / 2} \leqq 2(2 \delta d)^{1 / 2} .
$$

Hence

$$
\sup _{|x-y| \leqq \delta}\left|g_{c}^{1}(x)-g_{c}^{1}(y)\right| \leqq 2^{3 / 2}(\delta d)^{1 / 2}
$$

The proof is complete.

Definition. For $k$ a positive integer, let

$$
\alpha(k)=\mathcal{L}_{k}\left(E^{k} \cap \underset{z}{E}[|z| \leqq 1]\right) .
$$

TheOREM 2. If $c$ is a set of constant width in $E^{n}$, diam $c=d, k$ is a positive integer, $1<k<n$, and $\delta>0$, then

$\left(x \in E^{n-k}, y \in E^{n-k},|x-y| \leqq \delta\right)$

$$
\rightarrow\left(\left|g_{c}^{k}(x)-g_{c}^{k}(y)\right| \leqq 2^{3 / 2} \cdot(\delta d)^{1 / 2} \cdot \alpha(k-1) \cdot(d / 2)^{k-1}\right) .
$$

Proof. For $x \in E^{n-k}, t \in E^{k-1}$, define

$$
f_{x}(t)=g_{c}^{1}\left(x_{1}, \cdots, x_{n-k}, t_{1}, \cdots, t_{k-1}\right) .
$$

Then

$$
f_{x}(t)=\mathcal{L}_{1}\left(E^{1} \cap \underset{z}{E}\left[\left(x_{1}, \cdots, x_{n-k}, t_{1}, \cdots, t_{k-1}, z\right) \in c\right]\right) .
$$

Suppose now that $x \in E^{n-k}, y \in E^{n-k},|x-y| \leqq \delta$. Then

$$
\begin{aligned}
g_{c}^{k}(x)-g_{c}^{k}(y)= & \mathcal{L}_{k}\left(E^{k} \cap \underset{z}{E}\left[\left(x_{1}, \cdots, x_{n-k}, z_{1}, \cdots, z_{k}\right) \in c\right]\right) \\
& -\mathcal{L}_{k}\left(E^{k} \cap \underset{z}{E}\left[\left(y_{1}, \cdots, y_{n-k}, z_{1}, \cdots, z_{k}\right) \in c\right]\right) \\
= & \int_{E^{k-1}} f_{x}(t) d \mathcal{L}_{k-1} t-\int_{E^{k-1}} f_{y}(t) d \mathcal{L}_{k-1} t \\
= & \int_{E^{k-1}}\left(f_{x}(t)-f_{y}(t)\right) d \mathcal{L}_{k-1} t .
\end{aligned}
$$

Also, since $\mid\left(x_{1}, \cdots, x_{n-k}, t_{1}, \cdots, t_{k-1}\right)-\left(y_{1}, \cdots, y_{n-k}, t_{1}, \cdots\right.$, 
$\left.t_{k-1}\right)|=| x-y \mid \leqq \delta$, it follows by Theorem 1 that

$$
\begin{aligned}
\left|f_{x}(t)-f_{y}(t)\right|= & \mid g_{c}^{1}\left(x_{1}, \cdots, x_{n-k}, t_{1}, \cdots, t_{k-1}\right) \\
& -g_{c}^{1}\left(y_{1}, \cdots, y_{n-k}, t_{1}, \cdots, t_{k-1}\right) \mid \\
\leqq & 2^{3 / 2} \cdot(\delta d)^{1 / 2}, \quad \text { for } t \in E^{k-1} .
\end{aligned}
$$

Finally, let

$c^{\prime}=E^{k-1} \cap \underset{z}{E}\left[\left(w_{1}, \cdots, w_{n-k}, z_{1}, \cdots, z_{k-1}, w_{n-k+1}\right) \in c\right.$ for some $\left.w \in E^{n-k+1}\right]$.

Then diam $c^{\prime} \leqq d$ and

$$
\left(t \in\left(E^{k-1}-c^{\prime}\right)\right) \rightarrow\left(f_{x}(t)=f_{y}(t)=0\right) .
$$

Hence, applying the isodiametric inequality, we have

$$
\begin{aligned}
\left|g_{c}^{k}(x)-g_{c}^{k}(y)\right| & =\left|\int_{E^{k-1}}\left(f_{x}(t)-f_{y}(t)\right) d \mathcal{L}_{k-1} t\right| \\
& \leqq \int_{E^{k-1}}\left|f_{x}(t)-f_{y}(t)\right| d \mathcal{L}_{k-1} t \\
& =\int_{c^{\prime}}\left|f_{x}(t)-f_{y}(t)\right| d \mathcal{L}_{k-1} t \\
& \leqq 2^{3 / 2}(\delta d)^{1 / 2} \cdot \mathcal{L}_{k-1}\left(c^{\prime}\right) \\
& \leqq 2^{3 / 2}(\delta d)^{1 / 2} \cdot \alpha(k-1) \cdot(d / 2)^{k-1} .
\end{aligned}
$$

This completes the proof.

BROWN UNIVERSITY 